S. J. Y. Wang $\cdot$ S. Birtles $\cdot$ J. de Schoolmeester

J. Swales · G. Moody • D. Hislop · J. O'Dowd •

D. M. Smith · A. V. Turnbull - J. R. S. Arch

\title{
Inhibition of $11 \beta$-hydroxysteroid dehydrogenase type 1 reduces food intake and weight gain but maintains energy expenditure in diet-induced obese mice
}

Received: 27 October 2005 / Accepted: 27 January 2006 / Published online: 13 April 2006

C) Springer-Verlag 2006

\begin{abstract}
Aims/hypothesis: The 11 $\beta$-hydroxysteroid dehydrogenase type-1 inhibitor BVT.2733 lowers blood glucose and insulin in mutant mouse models of obesity and diabetes. Its effects on energy balance and body composition, and their contribution to improved glucose homeostasis have received little attention. Materials and methods: BVT.2733 $(100 \mathrm{mg} / \mathrm{kg}$, orally) was given twice daily to lean and diet-induced obese mice for 16 or 17 days. A group of obese mice was pair-fed to the amounts consumed by BVT.2733-treated mice. Results: In both obese and lean mice, BVT.2733 reduced food intake and weight gain, but increased water intake. Pair-feeding caused almost as great a decrease in body weight as BVT.2733. Energy expenditure was $38 \pm 8 \%$ higher in the BVT.2733-treated obese mice than in the pair-fed mice. Terminal plasma corticosterone was raised, lean body weight reduced and percentage fat unchanged in the pairfed mice (control, 47.8 $\pm 2.6 \%$; pair-fed, $47.1 \pm 1.9 \%$ ), whereas BVT.2733 did not reduce lean mass, but did reduce percentage fat $(40.9 \pm 2.0 \%)$. BVT.2733 but not pairfeeding reduced both the glucose tolerance AUC and the plasma insulin concentration $30 \mathrm{~min}$ after giving glucose. Conclusions/interpretation: BVT.2733 reduced food intake but prevented a concomitant reduction in lean body mass and energy expenditure. The latter effects may have contributed to improved glucose tolerance.
\end{abstract}

Keywords Body composition - Corticosterone - Dietinduced obesity - Energy expenditure - Energy intake Glucose tolerance $\cdot 11 \beta$-Hydroxysteroid dehydrogenase type-1 $\cdot$ Insulin $\cdot$ Metabolic syndrome $\cdot$ Mouse

\footnotetext{
S. J. Y. Wang · D. Hislop · J. O’Dowd · J. R. S. Arch $(\bowtie)$ Clore Laboratory, University of Buckingham,

Buckingham, MK18 1EG, UK

e-mail: jon.arch@buckingham.ac.uk

Tel.: +44-1280-820306

Fax: +44-1280-820135

S. Birtles · J. de Schoolmeester · J. Swales · G. Moody ·

D. M. Smith · A. V. Turnbull

Alderley Park, AstraZeneca,

Macclesfield, UK
}

Abbreviations HPA: hypothalamic-pituitary-adrenal HSD11B1: 11 $\beta$-hydroxysteroid dehydrogenase type-1

\section{Introduction}

$11 \beta$-Hydroxysteroid dehydrogenase type 1 (HSD11B1) catalyses the conversion of the inactive to active glucocorticoids. Inhibition of HSD11B1 is being pursued as an approach to the treatment of type 2 diabetes and visceral obesity. Since HSD11B1 primarily modulates levels of cortisol within tissues, it is hoped that its inhibition will not affect feedback inhibition of the hypothalamic-pituitaryadrenal (HPA) axis.

The selective inhibitor of murine HSD11B1, BVT.2733, lowered blood glucose and insulin in monogenic models of obesity and diabetes $[1,2]$. The anti-obesity activity of BVT.2733 has not, however, been addressed; nor has the possibility that its effects on energy balance contribute to its glucose-lowering effect. We therefore investigated whether and how BVT.2733 alters energy balance and body composition, and improves glucose tolerance in lean and diet-induced obese mice.

\section{Materials and methods}

3-Chloro-2-methyl- $N$-[4-[2-(4-methyl-1-piperazinyl)-2-oxoethyl]-1,3-thiazol-2-yl]benzenesulfonamide (BVT.2733) was dissolved in $0.5 \%$ hydroxypropylmethylcellulose $/ 0.1 \%$ Tween 80 .

Female C57B1/6 mice (Harlan Olac, Bicester, UK) were housed in threes and fed chow or, from the age of 16 weeks, a high-fat diet (by energy 68\% fat, $20 \%$ protein and $12 \%$ carbohydrate; $23.5 \mathrm{~kJ} / \mathrm{g}$; Charles River, Maidstone, UK). The lean and obese mice were 9 and 12 months old, respectively. They were dosed $(09.00$ and $17.00 \mathrm{~h})$ with BVT.2733 (100 mg/kg body weight, orally) or vehicle. A pair-fed group was given food at $09.00 \mathrm{~h}$.

Energy expenditure was measured by open-circuit indirect calorimetry, beginning at $11.00 \mathrm{~h}$ on day 11 . 
Intraperitoneal glucose $(1.5 \mathrm{~g} / \mathrm{kg})$ tolerance tests were conducted (days 15 or 16) after a 5-h fast. The mice were killed after being fasted for $5 \mathrm{~h}$ following their $09.00 \mathrm{~h}$ dose. Blood was taken from the neck for the measurement of plasma analytes. Body composition was measured using dual-energy X-ray absorptiometry (Lunar PIXImus twomouse densitometer and version 1.46 software; GE Medical, Bedford, UK).

Procedures were conducted in accordance with the University of Buckingham Home Office UK project licence under the Animals (Scientific Procedures) Act (1986) and as agreed by the University of Buckingham Ethical Review Board.

Statistical significance was evaluated by one-way ANOVA followed by Dunnett's test, or, where the BVT.2733 group was compared against both the control and pair-fed groups, Bonferroni's multiple-comparison test. Non-parametric Mann-Whitney $U$ tests were used for the insulin values for the lean mice because values in the treated mice fell below the limit of detection $(0.25 \mathrm{ng} /$ $\mathrm{ml})$. Results are given as means $\pm \mathrm{SE}$, except for the initial body weights given in the legend for Fig. $1 \mathrm{~b}$.

\section{Results}

BVT.2733 reduced food intake (Fig. 1a) and body weight (Fig. 1b). It increased water intake by $106 \%(p<0.05)$ in the lean and $66 \%(p<0.001)$ in the obese mice (results not shown). Pair-feeding caused almost as great a decrease in body weight as BVT.2733.

BVT.2733 increased energy expenditure $(p<0.001)$ by $38 \%(\mathrm{~kJ} /$ mouse) or $33 \%(\mathrm{~kJ} / \mathrm{g}$ mouse) compared with the pair-fed mice (Fig. 1c). The difference between the two groups was due to energy expenditure being suppressed in pair-fed mice between 17.00 and $11.00 \mathrm{~h}$ but tending to be elevated over $24 \mathrm{~h}$ in the BVT-2733-treated mice.

The pair-fed obese mice tended to have a reduced body fat mass (Fig. 1d), but since lean mass was also reduced (Fig. 1e), there was no effect on percentage fat content (Fig. 1f). By contrast, BVT-2733 tended to reduce body fat content and increase lean mass in the obese mice; consequently it reduced percentage body fat. BVT.2733 reduced both absolute and percentage body fat in the lean mice.

Prior to the glucose tolerance test, BVT.2733 $(p<0.05)$ and pair-feeding $(p<0.001)$ reduced the fasting glucose level in the obese mice; BVT.2733 had significantly $(p<0.001)$ less effect than pair-feeding (Fig. 2a). However, BVT.2733, but not pair-feeding, improved glucose tolerance in the obese mice. BVT.2733 also improved glucose tolerance in the lean mice (Fig. 2a,b).

BVT.2733 but not pair-feeding reduced post-glucose insulin in the obese mice. BVT.2733 also reduced plasma insulin in the lean mice (Fig. 2c). At termination, fasting insulin was reduced in the BVT.2733-treated mice compared with the pair-fed obese mice (obese control,

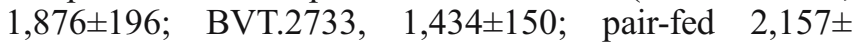
$283 \mathrm{pg} / \mathrm{ml})$.
The plasma NEFA concentration was lower in the BVT.2733-treated mice than in the pair-fed mice (Fig. 2d). In fed mice on the 12th day, BVT.2733 reduced the plasma triacylglycerol concentration relative to the obese control and especially to the pair-fed group (Fig. 2e). Similar results were obtained at termination in fasted mice. The terminal plasma corticosterone concentration was elevated in the pair-fed group relative to both the obese control and the BVT.2733-treated group (Fig. 2f).

\section{Discussion}

Although visceral obesity is a feature of the metabolic syndrome, the anti-obesity activity of BVT.2733 was not addressed in previous short studies in monogenic models of obesity and diabetes $[1,2]$. Another selective HSD11B1 inhibitor also reduces body weight and food intake in dietinduced obese mice [3], but the contribution of altered food intake and energy expenditure to its other activities was not investigated.

The effects of BVT.2733 on energy balance, body composition and glucose homeostasis in obese mice were reinforced by similar findings in lean mice. However, a number of effects of BVT.2733 in obese mice could not be explained by reduced food intake. Pair-feeding but not BVT.2733 reduced the lean tissue content and increased the terminal plasma corticosterone concentration of the obese mice, while BVT.2733 but not pair-feeding reduced their percentage fat content. Energy expenditure was reduced by pair-feeding but not by BVT.2733. Deletion of the gene encoding HSD11B1 also affects energy expenditure, since it results in resistance to diet-induced obesity despite increased energy intake [4].

BVT.2733, but not pair-feeding, improved glucose tolerance in the diet-induced and, by contrast with a previous study [2], lean mice. Only BVT.2733 reduced the plasma insulin concentration during the glucose tolerance test, and the terminal NEFA concentration was reduced relative to the pair-fed mice, consistent with improved insulin sensitivity.

The reduction in food intake in response to BVT.2733 must ultimately involve a central signal. It could have been initiated by inhibition of HSD11B1 in the periphery, which, by reducing corticosterone regeneration, enhanced its metabolic clearance, decreased feedback inhibition of the HPA axis, increased the release of corticotrophin-releasing hormone and reduced food intake [5]. However, BVT.2733 did not reduce the terminal corticosterone concentration. Alternatively, direct inhibition of HSD11B1 in the hippocampus, hypothalamus or anterior pituitary may have reduced feedback inhibition of the HPA axis with the same consequences.

By contrast with food intake, inhibition of HSD11B1 in the periphery might increase energy expenditure without affecting plasma corticosterone. However, most evidence suggests that energy expenditure in rodents is increased by glucocorticoid antagonism due to a reduction in central feedback inhibition of the HPA axis. The resulting increase 
a
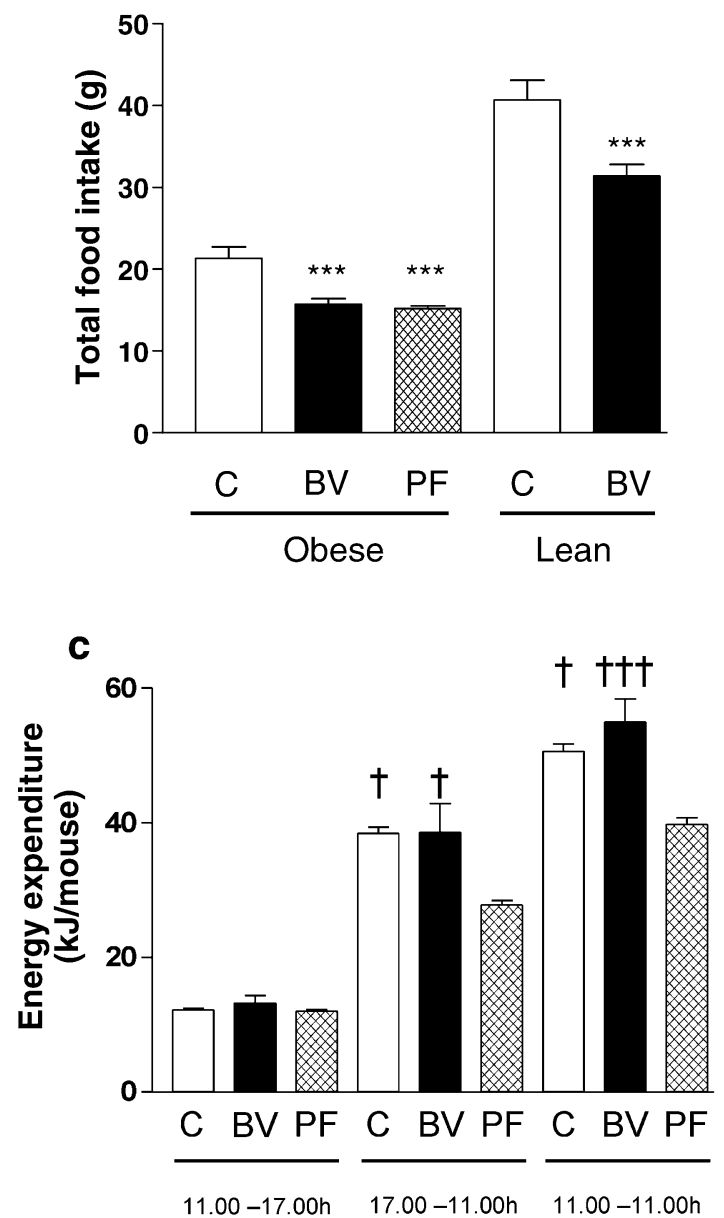

e

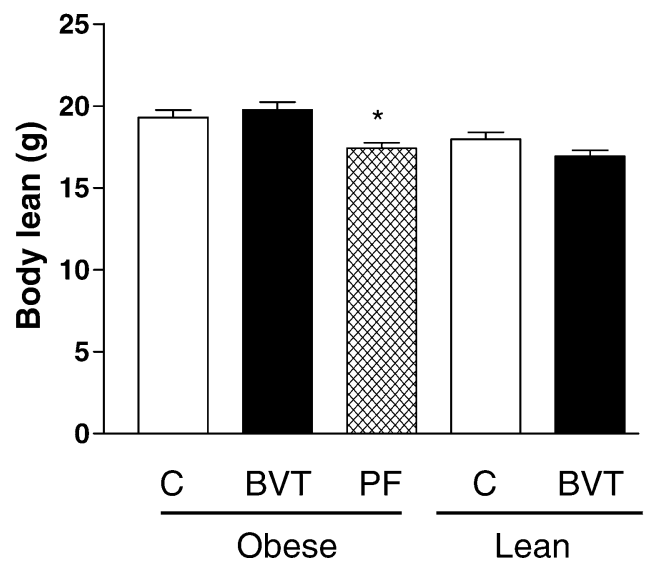

b

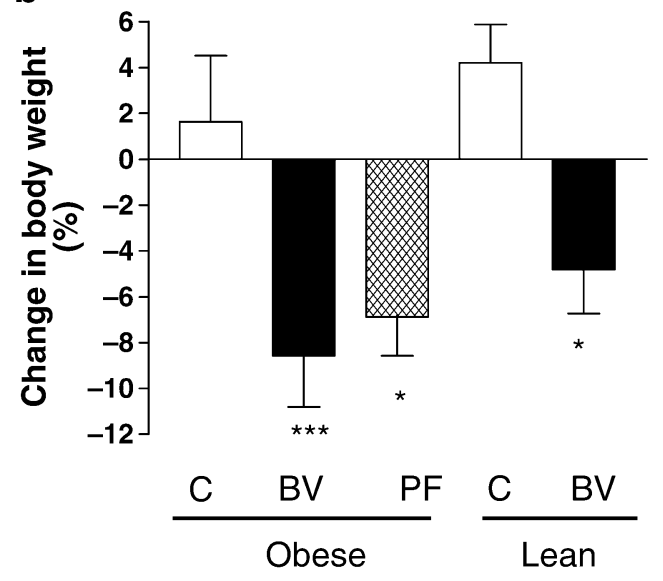

d

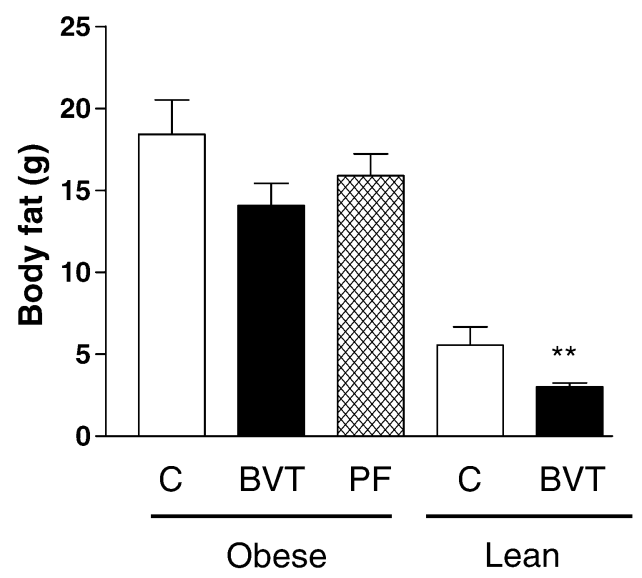

f

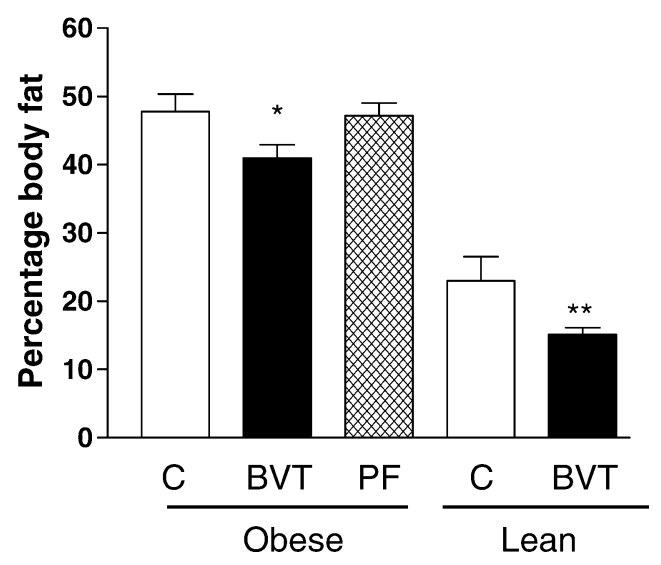

Fig. 1 Energy balance and body composition in control $(C)$, BVT.2733-treated $(B V)$ and pair-fed $(P F)$ mice. a Food intake over 16 days. b Percentage change in body weight. Initial body weights (g) were: obese, 39.1 $\pm 6.2, n=30$; lean, 24.0 $\pm 1.3, n=12$ (means \pm SD). c Energy expenditure in obese mice for the periods 11.00 $17.00 \mathrm{~h}$ (light period prior to dosing at $17.00 \mathrm{~h}$ ), 17.00-11.00 $\mathrm{h}$ and the complete 24-h period. d Body fat mass at termination. e Body lean mass. f Percentage body fat. For body weight and composition, $n=9$ or 12 (BVT.2733 group) for obese mice and $n=6$ for lean mice; for food intake, $n=3$ or $n=4$ for obese mice and $n=2$ for lean mice; for energy expenditure, $n=3$. ${ }^{*} p<0.05$; $* * p<0.01$; $* * * p<0.001$ compared with obese or lean control group. ${ }^{*} p<0.05$; ${ }^{\dagger \dagger} p<0.001$ compared with the pair-fed group. Bars are SEs (for food intake in lean mice where $n=2$, the bar also indicates the higher of the two values) 
a

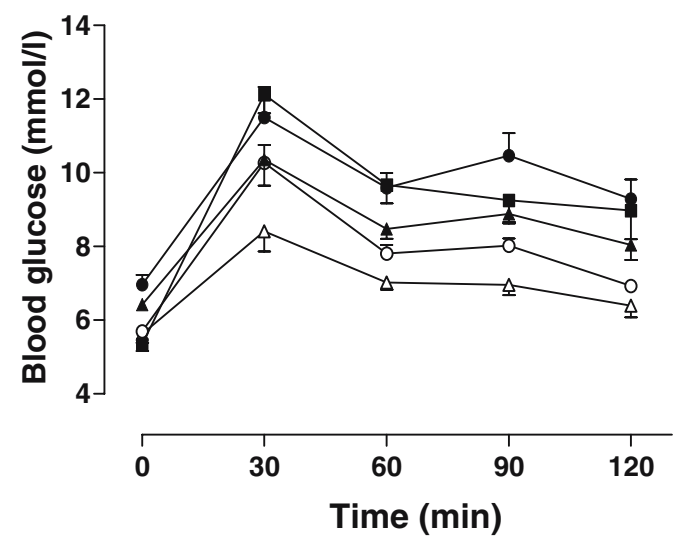

C

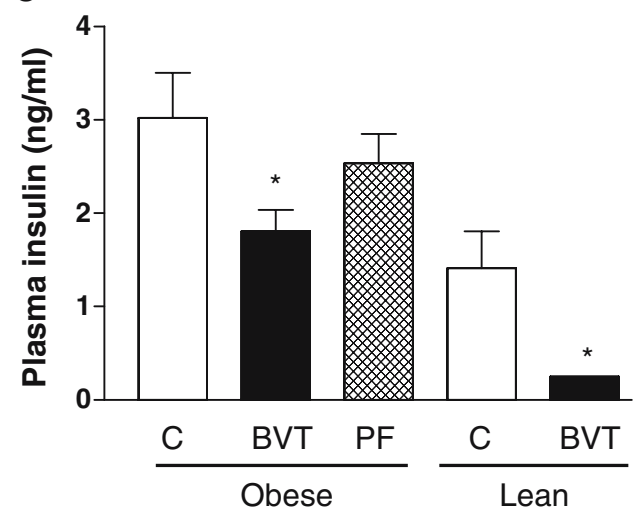

e

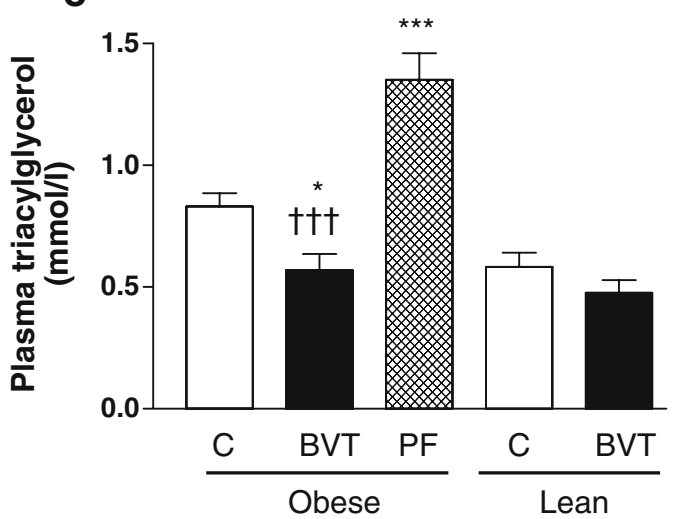

Fig. 2 Glucose tolerance test and plasma analytes in control $(C)$, BVT.2733-treated $(B V)$ and pair-fed $(P F)$ mice. a Blood glucose immediately before $(0 \mathrm{~min})$ and at times after giving i.p. glucose to control (circles), BVT.2733-treated (triangles) and pair-fed (squares) obese (closed symbols) and lean (open symbols) mice. b Total AUCs from a. c Plasma insulin $30 \mathrm{~min}$ after giving

in corticotrophin-releasing-factor activity not only decreases food intake but also increases sympathetic activity [6]. In the present study, BVT.2733 may have prevented a reduction in sympathetic activity in response to reduced energy intake by preventing an increase in plasma corticosterone (Fig. 2f). Altered activities of the HPA axis and sympathetic nervous system may also account for the b

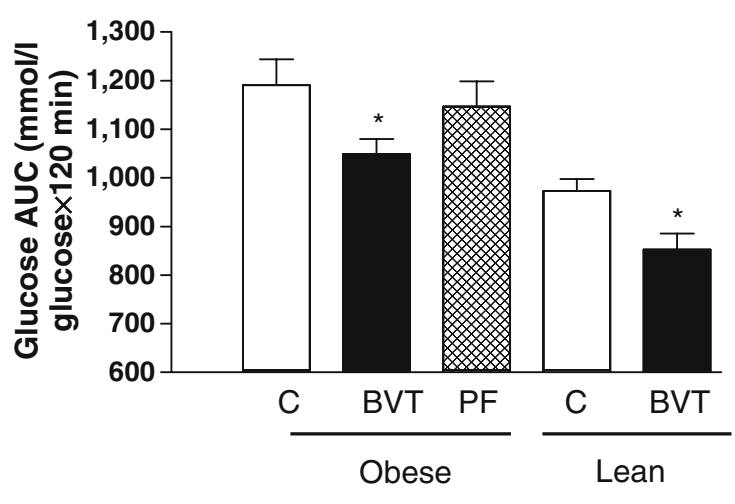

d

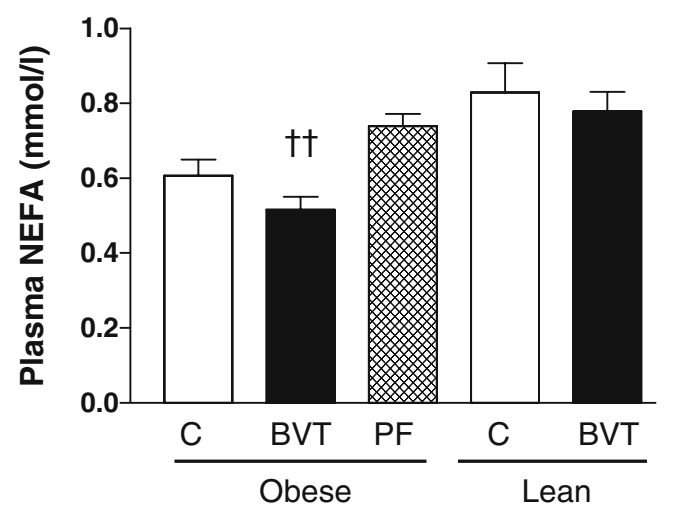

f

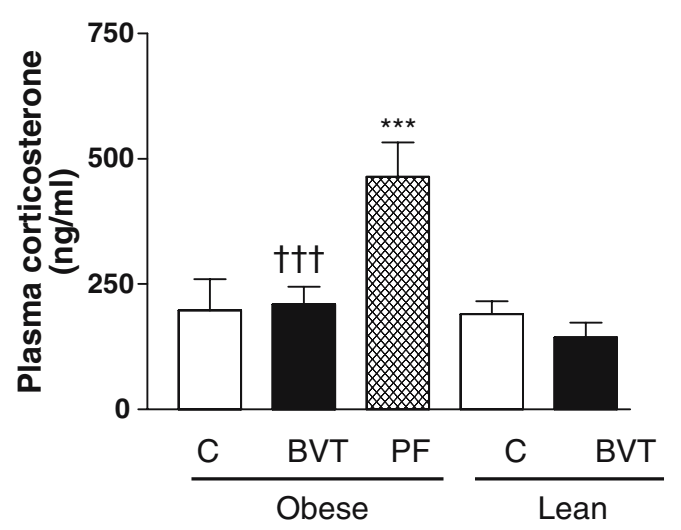

glucose. d Terminal plasma NEFA; e triacylglycerol in fed mice on day $12 ; \mathbf{f}$ terminal plasma corticosterone. $n=9$ for obese and 6 for lean mice. ${ }^{*} p<0.05 ; * * * p<0.001$ compared with obese or lean control group. ${ }^{\dagger} p<0.01 ;{ }^{\dagger} p<0.001$ compared with the pair-fed group

finding that pair-feeding reduced the lean tissue content of the obese mice, whereas BVT.2733 tended to increase lean and decrease fat, with the result that percentage body fat was reduced. Thus restriction of energy intake in rodents or humans causes loss of lean tissue as well as fat, whereas $\beta_{3}$-adrenoceptor agonists, which mimic the activity of the 
sympathetic nervous system, cause selective loss of fat and may even increase lean tissue [7].

A direct effect of BVT.2733 on insulin sensitivity in peripheral tissues due to their reduced exposure to corticosterone would be expected. The effect of BVT.2733 on glucose tolerance does not appear to have been due to reduced food intake because pair-feeding was ineffective. However, prevention of reduced lipid oxidation and energy expenditure in response to reduced energy intake could have played a role [8], as could maintenance of lean body mass.

The role of increased energy expenditure in the antiobesity and insulin-sensitising actions of HSD11B1 inhibitors may be important because it is unclear whether an HSD11B1 inhibitor would also increase energy expenditure in humans. Cortisol and methyl prednisolone have both been found to increase rather than decrease resting energy expenditure in humans, while the glucocorticoid receptor antagonist mifepristone (RU486) had no effect [9]. In any event, it will be important to establish whether HSD11B1 inhibitors affect sympathetic activity in humans.

Increased water intake in response to BVT.2733 raises the possibility that it had a diuretic effect. However, it seems unlikely that diuresis could be due to decreased stimulation of the kidney mineralocorticoid receptor by corticosterone because HSD11B1 is not produced in cells that express the mineralocorticoid receptor [10].

In conclusion, an HSD11B1 inhibitor has an anti-obesity activity that was not associated with reduced lean body mass or energy expenditure. Improved glucose tolerance may have been at least partly due to these effects.

Acknowledgements We thank A. Roberts, E. Wargent and B. Brown for technical support, and M. Cawthorne for advice at all stages.

Duality of interest The work of S. J. Y. Wang at the University of Buckingham was funded by AstraZeneca. He is now employed by AstraZeneca. He was supervised by J. R. S. Arch, who received no payment for this supervision but is an ad hoc consultant for AstraZeneca. Six authors were employed by AstraZeneca when this work was being performed. AstraZeneca has an interest in inhibitors of HSD11B1, but has no such agent in clinical development.

\section{References}

1. Alberts P, Engblom L, Edling N et al (2002) Selective inhibition of 11 beta-hydroxysteroid dehydrogenase type 1 decreases blood glucose concentrations in hyperglycaemic mice. Diabetologia 45:1528-1532

2. Alberts P, Nilsson C, Selen G et al (2003) Selective inhibition of 11 beta-hydroxysteroid dehydrogenase type 1 improves hepatic insulin sensitivity in hyperglycemic mice strains. Endocrinology 144:4755-4762

3. Hermanowski-Vosatka A, Balkovec JM, Cheng K et al (2005) 11beta-HSD1 inhibition ameliorates metabolic syndrome and prevents progression of atherosclerosis in mice. J Exp Med 202:517-527

4. Morton NM, Paterson JM, Masuzaki H et al (2004) Novel adipose tissue-mediated resistance to diet-induced visceral obesity in 11 beta-hydroxysteroid dehydrogenase type 1-deficient mice. Diabetes 53:931-938

5. Zorrilla EP, Tache Y, Koob GF (2003) Nibbling at CRF receptor control of feeding and gastrocolonic motility. Trends Pharmacol Sci 24:421-427

6. Richard D, Huang Q, Timofeeva E (2000) The corticotropinreleasing hormone system in the regulation of energy balance in obesity. Int J Obes Relat Metab Disord 24 [Suppl 2]:S36-S39

7. Arch JR (2002) $\beta_{3}$-Adrenoceptor agonists: potential, pitfalls and progress. Eur J Pharmacol 440:99-107

8. Hulver MW, Dohm GL (2004) The molecular mechanism linking muscle fat accumulation to insulin resistance. Proc Nutr Soc 63:375-380

9. Jobin N, de Jonge L, Garrel DR (1996) Effects of RU 486 on energy expenditure and meal tolerance in normal men. $\mathrm{J}$ Am Coll Nutr 15:283-288

10. Stewart PM, Krozowski ZS (1999) 11ß-Hydroxysteroid dehydrogenase. Vitam Horm 57:249-324 